This item was submitted to Loughborough's Research Repository by the author.

Items in Figshare are protected by copyright, with all rights reserved, unless otherwise indicated.

\title{
Parametric conditions for generation of stable atmospheric pressure nonthermal plasmas
}

PLEASE CITE THE PUBLISHED VERSION

PUBLISHER

(c) IEEE

VERSION

VoR (Version of Record)

LICENCE

CC BY-NC-ND 4.0

\section{REPOSITORY RECORD}

Deng, Xu-Tao, and Michael G. Kong. 2019. "Parametric Conditions for Generation of Stable Atmospheric Pressure Nonthermal Plasmas". figshare. https://hdl.handle.net/2134/5232. 
This item was submitted to Loughborough's Institutional Repository (https://dspace.lboro.ac.uk/) by the author and is made available under the following Creative Commons Licence conditions.

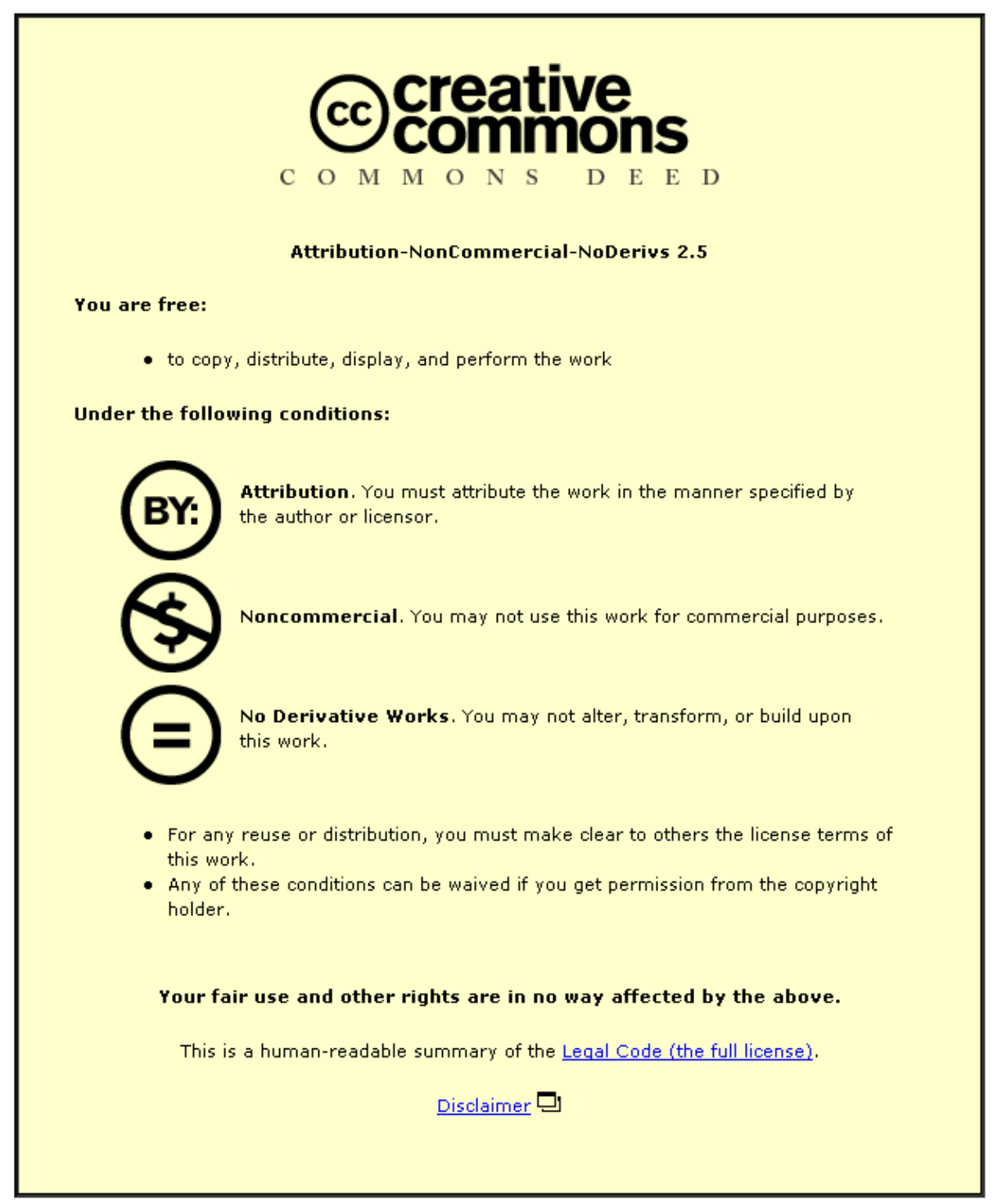

For the full text of this licence, please go to: http://creativecommons.org/licenses/by-nc-nd/2.5/ 


\title{
Parametric Conditions for Generation of Stable Atmospheric Pressure Nonthermal Plasmas
}

\author{
$X \mathrm{~T}$ Deng and $\mathrm{M} \mathrm{G}$ Kong \\ Department of Electronic and Electrical Engineering \\ Loughborough University, Leicestershire LE11 3TU, UK
}

\begin{abstract}
Nonthermal plasmas generated at atmospheric pressure have recently found many applications such as surface cleaning and pollution control. One plasma generation configuration consists of two dielectrically insulated parallel plates with application of an $\mathrm{AC}$ voltage of several KV and several $\mathrm{kHz}$. Such a plasma generating structure is very similar to many $\mathrm{HV}$ insulating systems and equipment, and as such they share much similarity in the evolving plasma and associated plasma-structure interaction. In this contribution, we develop a one-dimensional numerical model to simulate discharge plasmas supported by such insulated electrode structure and for simplicity we consider helium as the working gas. Assuming that the hydrodynamic approximation applies, various ionization, excitation, and recombination processes are considered as sources of generation of charged particles and excited species. Boltzmman equations are then solved in conjunction with Poisson equation. Numerical examples are then used to explore the conditions under which the induced plasma is nonthermal. Also studied is dependence of the plasma upon frequency of the applied voltage and the pressure of the ambient gas. These correlative relationships should also be true for many insulating devices and systems.
\end{abstract}

\section{Introduction}

Gas discharges generated at atmospheric pressure are common in a number of high voltage equipment, devices, and systems, for instance on surfaces of laminated insulators and in microscopic cavities of bulk insulators. In the majority of the cases, these discharge plasmas are undesirable as they compromise the integrity of the insulator sub-system and hence the overall high-voltage system. There are also cases where these discharge plasmas are encouraged for instance when used to control pollutants such as volatile organic components (VOCs). It is therefore both useful and important to study their behaviours to understand their effects and to explore ways to control them.
In the context where these gas discharges are generated deliberately, such as for VOC control, there has been interest to develop numerical tools for their studies. In principle, the basic methodology can also be applied generically to cases where the generation of these gas plasmas is undesirable. This has motivated us to develop a generic numerical model for studies of atmospheric pressure gas discharges for both of the above-mentioned practical situations. One challenge for this generic approach is that the electric excitation for the gas discharge cannot be defined very well, in terms of its peak voltage and its spectral details. In addition these gas discharges can be thermal, nonthermal, or both during their life. It is both unrealistic and unnecessary to cater for such a wide range of eventualities within a single physical and numerical framework.

As a first step, we assume that the electric excitation is an ac voltage of several $\mathrm{kV}$ and several $\mathrm{kHz}$. With a successful develop of relevant numerical codes under this simplified conditions, we will develop more sophisticated models for more generalised excitation conditions. In terms of the basic plasma characteristics of these gas discharges, we note that in many practical cases they are not particularly energised because of many safety precautions imposed to restrict their growth. Hence it is reasonable to assume them to be nonthermal. Furthermore the ambient gas will be assumed as helium as a simplification rather than the more practical air.

In this paper, we will first describe a simple onedimensional model for an ac excited atmospheric discharge generated in pure helium. We will then use the numerical model developed to study several numerical cases. In particular, we will consider the minimum electron density for the generated discharge to be stable through many cycles of the applied electric field. This is an useful and practical indicator of possible measures needed to either restrict or encourage such gas discharges. 


\section{The discharge model}

The excitation configuration for the gas plasma consists of two parallel plates, each coated with a dielectric layer and connected externally to an ac voltage source. The background gas is pure helium at atmospheric pressure. The ionised helium is treated as a fluid whose dynamics are governed by the Boltzmann's equations. Under the hydrodynamic assumptions, the latter result in the electron and ion continuity and momentum transfer equations. This facilitates a macroscopic description of the plasma dynamics without its microscopic details. The electric field in the space between the two parallel plates is calculated from Poisson's equation. These two sets of equations are coupled together via electron and ion densities. Specifically the mathematical equations for the discussed problem are given below

$\frac{\partial n_{x}}{\partial t}=S_{x}(\bar{r}, t)-\frac{\partial\left[n_{x}(\bar{r}, t) W_{x}(\bar{r}, t)\right]}{\partial \vec{r}}+\frac{\partial^{2}\left[n_{x}(\bar{r}, t) D_{x}(\bar{r}, t)\right]}{\partial \bar{r}^{2}}$

$\frac{\partial E_{d s}(\bar{r}, t)}{\partial r}=\frac{|e|}{\varepsilon_{0}}\left[n_{+}(\vec{r}, t)-n_{-}(r, t)\right]$

where $S_{\mathrm{x}}$ is the source term, $n_{+}$and $n$. are the ion and electron densities respectively. $D_{\mathrm{x}}$ and $W_{\mathrm{x}}$ are diffusion coefficient and drift velocity respectively. Other relevant reaction coefficients, including ionisation coefficients, are largely based on [1][2]. Particular attentions need to be paid to the choice of boundary conditions. Numerical algorithm used to solve the above equations is essentially the Pantankar scheme[3].

\section{Conditions of stable discharges}

For all numerical examples considered here, the electrode capacitance is $70 \mathrm{pF}$. The two electrodes have a separation gap of $0.5 \mathrm{~cm}$ and a radius of $2 \mathrm{~cm}$. The external voltage is of $1.6 \mathrm{kV}$ peak and $10 \mathrm{kHz}$ in frequency. Initially the gas voltage is $950 \mathrm{~V}$ though initial voltage of the electrodes forming capacitor. Gas temperature is assumed at $293.16 \mathrm{~K}$, and the gas breakdown voltage is assumed to be $1.1 \mathrm{kV}$.

Through numerical studies, it has been found that to obtain temporarily stable discharge it is necessary to set its recombination rate at $40 \times 10^{-6} \mathrm{~cm}^{-3} / \mathrm{s}$, much greater than what has been reported in literature. While this empirically arrived figure needs to be justified, and its value is similar to that used in a recent simulation of nonthermal atmospheric plasmas[1]. Possible causes for this much elevated recombination rate may include the high frequency of the applied excitation voltage. We also note that electrons reaching the electrode surface tend to be absorbed there either through capacitor charging or through microscopic attachment resulting from negative affinity of the electrode coating materials. Therefore we introduce an electron absorption coefficient, and typically we assume it between 0 and 0.01 . Numerical calculation suggests that the choice of the electron absorption coefficient is not as crucial as the recombination coefficient.

Figure 1 shows a plot of gas voltage, discharge current, and applied voltage as a function time obtained with an initial electron density of $5 \times 10^{6} \mathrm{~cm}^{-3}$. It is clear that the discharge current is almost identical through the first five cycles of the applied voltage. Further numerical computations suggest that this remains unchanged through many subsequent cycles and so the

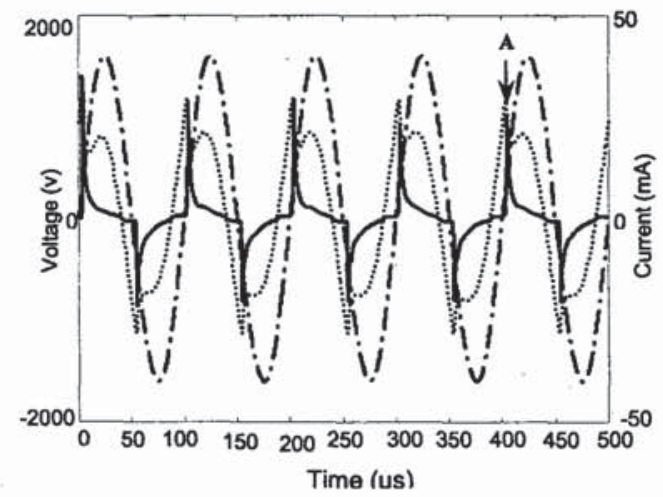

Figure 1: Time dependence of the applied voltage (dot-dashed curve), gas voltage (dashed curve), and discharge current (solid curve).

discharge plasma is stable repeating the same characteristics throughout a long period of time.

In figure 2, spatial distributions of electric field, ion density, and electron density across the electrode gap are shown. These resemble, to certain extent, to the spatial characteristics of a dc low-pressure glow discharge. However the ion and electron densities are much lower than those obtained experimentally[1], by approximately two magnitudes. Qualitatively this is understandable because impurities are known to play a very significant role in this type of gas discharges and they tend enhance ionisation[1]. Effects of impurities will be considered in our numerical model and reported in a future note.

It is interesting to explore parametric conditions for stable gas discharges. For instance, a reduction in the electron absorption at the electrode surface from 0.01 to 0 would generally lead to a less significant discharge with a smaller discharge current. It is also possible under this reduced electron absorption that the gas discharge may die out gradually. This may be resulted from an accumulated mismatch of the dielectric voltage (the residual voltage on the electrodes' dielectric 
coating) to the applied voltage. Nevertheless it does not appear that it is crucial to use precise value for the

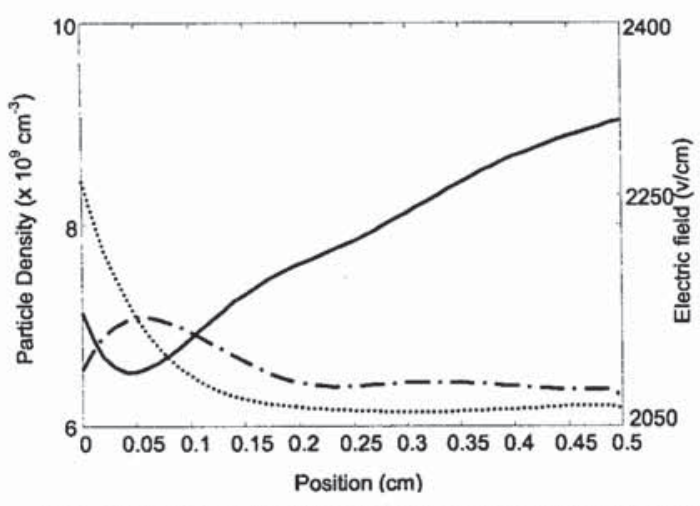

Figure 2: Spatial distribution of electric field (solid curve), helium ion density (dotted curve), and electron density (dot-dashed curve).

absorption rate.

It is also interesting to see whether stable discharges can be obtained if the excitation frequency alters. With all other system parameters remained unchanged, an increase in the excitation frequency from $10 \mathrm{kHz}$ to $100 \mathrm{kHz}$ is found to unstable the gas discharge within the first cycle of the applied voltage. In this case, the gas plasma is likely o extinguish very rapidly. However if the excitation frequency is increase further to $1 \mathrm{MHz}$, a more stable discharge re-appears, repeatable through the first $5-10$ cycles at least although this high frequency discharge has a distinctively different temporal feature from the $10 \mathrm{kHz}$ discharge. This may not necessarily suggest that it is less likely to produce a stable discharge at higher frequencies. Instead this suggests that other parametric conditions may need to be re-adjusted in order to introduce the required stability into the gas discharge generated.

\section{Effects of initial electron density}

The above numerical examples are obtained with an initial electron density of $5 \times 10^{6} \mathrm{~cm}^{-3}$. This is an important system parameter. An understanding of the minimum initial electron density at a given excitation frequency provides an indication of the necessary repetition rate of subsequent discharge events to maintain the stability of the gas discharge. A smaller repetition rate would lead to a reduced gas ionisation through less frequent electron-neural collisions, and as such to a gradual extinction of the initial gas discharges. On the other hand, a greater repetition rate leads to a more significant gas ionisation and so has the danger to overheating the gas plasma.

To this end, effects of initial electron density have been studied under the otherwise identical parametric conditions. It has been found that different initial electron densities result in different temporal profiles in voltage and current of the generated discharge. However this difference is not significant, and does not affect the generation of stable gas discharges. Also it becomes smaller as the initial electron density becomes greater. This is likely due to the fact that a discharge with a small initial electron density needs a longer time to settle into its steady state. It is also interesting that discharges of different initial electron densities become very similar after the excitation voltage is applied for sufficiently long period of time (after first $10-20$ cycles). This suggests that the generation of stable atmospheric plasmas is reasonably robust, relatively independent of the initial electron density.

Figure 3 illustrates the gas voltage and discharge current as a function time when the initial electron density is reduced to $1 \mathrm{~cm}^{-3}$. Continuation of numerical computation into subsequent cycles of the applied voltage shows that it is a stable discharge. Figure 4 shows that the peak electron density averaged over the first 10 cycles of the applied voltage as a function of the initial electron density. It is clear that the electron density of a settled, steady-state atmospheric plasma is relatively independent of the initial electron density

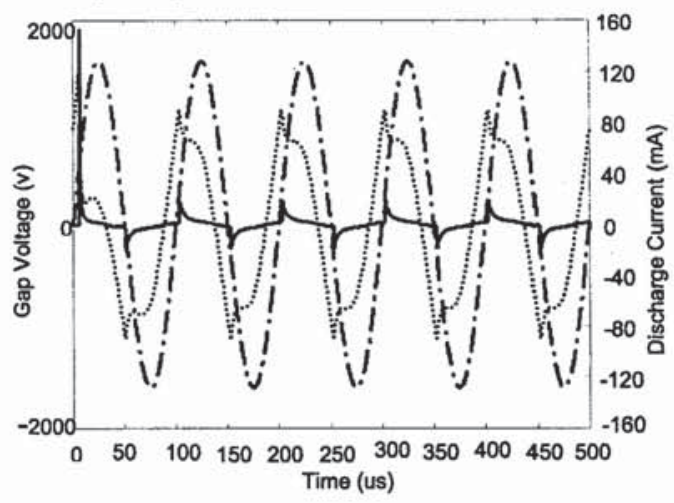

Figure 3: Applied voltage (dot-dashed curve), discharge current (solid curve), and gas voltage (dot curve) for an initial electron density of $1 \mathrm{~cm}^{-3}$.

used. It should be mentioned that when the initial

electron density becomes greater than $10^{11} \mathrm{~cm}^{-3}$ the stability of these atmospheric plasmas is found to break down. 


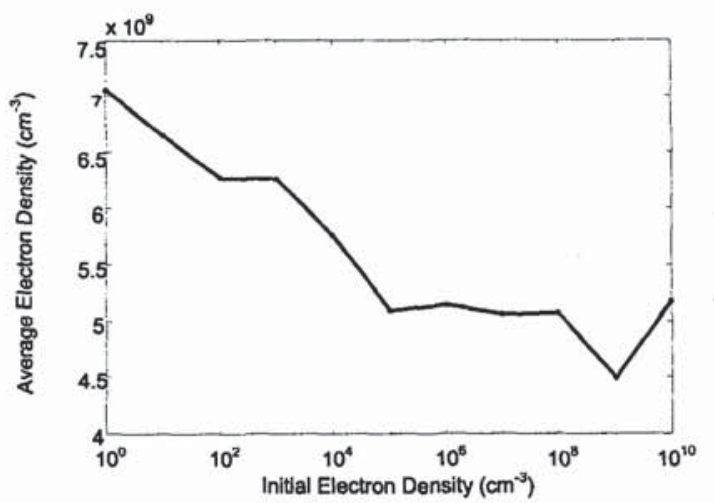

Figure 4: Peak electron density averaged over the first 10 cycles of the applied voltage against the initial electron density.

\section{Conclusion}

In this contribution, a numerical code is developed to study parametric conditions to generate stable lowpower atmospheric pressure discharges in pure helium. It was found that this depends crucially on the choice of the recombination rate and to a lesser extent on that of the electron absorption rate at electrode surfaces. It was also found that stable generation of these atmospheric plasmas can be achieved relatively independent of the initial electron density between $1 \mathrm{~cm}^{-3}$ to $10^{11} \mathrm{~cm}^{-3}$. However when the initial electron density becomes great than $10^{11} \mathrm{~cm}^{-3}$, these atmospheric plasmas become highly unstable and extinguish within the first two cycles of the applied voltage.

In terms of reaction coefficients, it was found that both the recombination rate and the electron absorption rate are important to influence the stability of the generated atmospheric gas discharges, in particular the recombination rate. A much increased recombination rate from usually quoted figures in literature was found necessary to stabilise these atmospheric plasmas. These findings are useful in providing insights into the fundamental processes in these atmospheric plasmas.

\section{Reference:}

[1] F. Massines, A. Rabehi, Ph. Decomps, R. B. Gadri, P. Segur, and C. Mayoux, "Mechanisms of a glow discharge at atmospheric pressure controlled by dielectric barrier", Journal of Applied Physics, vol. 83 , no.6, pp. $2950-2957,1998$.
[2] A. L Ward, "Calculations of cathode-fall characteristics", Journal of Applied Physics, vol. 33, no.9, pp. 2789-2794, 1964.

[3] S. Patankar, "Numerical heat transfer and fluid flow", Hemisphere publishing corporation, 1980. 